\title{
How Stress and Mental Workload are Connected
}

\author{
Norah H. Alsuraykh \\ School of Computer Science \\ University of Nottingham \\ Nottingham, UK \\ norah.alsuraykh@nottingham.ac.uk
}

\author{
\begin{tabular}{c} 
Max L. Wilson \\
School of Computer Science \\
University of Nottingham \\
Nottingham, UK \\
max.wilson@nottingham.ac.uk \\
\hline
\end{tabular} \\ \begin{tabular}{c} 
Max L. Wilson \\
School of Computer Science \\
University of Nottingham \\
Nottingham, UK \\
max.wilson@nottingham.ac.uk \\
\hline
\end{tabular} \\ \begin{tabular}{c} 
Max L. Wilson \\
School of Computer Science \\
University of Nottingham \\
Nottingham, UK \\
max.wilson@nottingham.ac.uk \\
\hline
\end{tabular} \\ \begin{tabular}{c} 
Max L. Wilson \\
School of Computer Science \\
University of Nottingham \\
Nottingham, UK \\
max.wilson@nottingham.ac.uk \\
\hline
\end{tabular} \\ \begin{tabular}{c} 
Max L. Wilson \\
School of Computer Science \\
University of Nottingham \\
Nottingham, UK \\
max.wilson@nottingham.ac.uk \\
\hline
\end{tabular}
}

Sarah Sharples

Faculty of Engineering

University of Nottingham

Nottingham, UK

sarah.sharples@nottingham.ac.uk

\author{
Paul Tennent \\ School of Computer Science \\ University of Nottingham \\ Nottingham, UK \\ paul.tennent@nottingham.ac.uk
}

\begin{abstract}
Mental Workload (MWL) can be both good and bad; we can thrive under high MWL, or our performance can drop if the demands become either too low or too high. Similarly, stress is not always bad, short term stress can be beneficial to overcome a challenge or dangerous situation. In our research, we have seen both people that enjoy high workload, and people that feel stressed by it, but we do not know whether that experience of stress significantly affects our measurements. Our recent results show that fNIRS measurements are affected by stress (measured by SSSQ). This paper seeks to discuss the relationship between these concepts, discussing examples of where similar influencing factors appear within models of both Stress and Mental Workload, as well as within subjective measures of them. We conclude that future work must consider participants' experiences of both Stress and Mental Workload, as well as other cognitive concepts, when trying to estimate them from physiological measures.
\end{abstract}

\section{KEYWORDS}

Stress; Mental Workload; Anxiety; SSSQ; NASA-TLX; MIST;

\section{Introduction}

Recently, Human Computer Interaction (HCI) researchers have focused on using measures of physiological changes to estimate different cognitive activities, such as Stress using e.g. skin response [26] or brain data [56]. Many studies, in fact, show that these types of sensors can pick up different types of cognitive or emotional reactions. Some have pointed out that an increase in Heart Rate is linked with high mental workload [9] [52] while others show that increase in Heart Rate is also triggered by stress [10] [15] [46], and surprise [40], or indeed just physical movement.
Our own research, and research from others in the HCI community, has focused on fNIRS as movement-tolerant brain sensor for use in HCI experiments [27] [33] [37] [44]. For example, it has been used to evaluate the UX of software [34] and used to adapt tasks [1] [56]. While using fNIRS to measure Mental Workload during Air Traffic Control (ATC) tasks, however [38] noted that some people found the task stressful while some found it enjoyable.

Our current studies have been attempting to understand how to measure the impact of stress on fNIRS measurements of Mental Workload, and visa versa. While there are numerous studies which have used fNIRS to discover MWL and Stress separately, few researchers have examined the experiences of stress with MWL and whether this experience of stress is affecting the MWL and physiological measurements or not. We used, for example, the Montreal Imaging Stress Task (MIST) to manipulate stress (in the form of time pressure and negative feedback) whilst maintaining task demand (mathematical difficulty). Our recent NASA-TLX results, however, also showed that mental workload was increased at the same time as stress.

The challenge of manipulating stress and mental workload separately has led us to consider more closely the relationship between them. Interestingly, it is broadly accepted and noted in literature that some people enjoy high workload and believe that it helps them to be more productive, whereas others feel stressed by high-workload and pressure and are less productive. The relationship between MWL and stress is much more complicated than: high workload is bad, and indeed that: experienced stress is bad. As there is a lack of clarity in how stress and MWL are related, because they are used to describe often similar phenomena, this paper reviews literature to clarify the relationship between these two concepts, and elaborate on why they are hard to study separately. 


\section{Background}

\subsection{Mental Workload}

Mental workload (MWL) could affect the individuals who are interacting with computers and other devices while navigating complex interfaces that might impose high cognitive demands. Mental overload may compromise a user's performance, by increasing error rates and decline in motivation [54]. MWL has been described as "the relationship between primary task performance and the resources demanded by the primary task" [52]. In other words, MWL is the relationship between demands placed upon individuals and their capacity to cope with it. Many measures for assessing mental workload have been developed and they are classified into three types: performance-based assessment, subjective workload assessment, and physiological workload assessment [53]. Traditionally, the effect of MWL is self-reported by the individual using well-established and formally validated subjective techniques such as the NASA Task Load Index (NASATLX) retrospectively after a task see e.g. [38] or Instantaneous SelfAssessment of workload technique (ISA) concurrently with a task [25]. In the case of overload, users may even stop reporting ISA scores, because they lack the spare capacity to complete this secondary task. There are measurable physiological changes, however, that correlated consistently with high MWL: facial temperature with our nose temperature dropping with higher mental workload levels [11], pupil diameter [5]

Much HCI research is performed on the premise that high MWL is bad, while [53] highlight that a decrease in performance could also be the result of underload conditions. For underload, the concern is that demand is so low for an extended period of time, that participants' attention to a task also reduces. Conversely, high MWL, may be recognized as desirable, such as in cases where people are driven in high-pressure jobs, enjoy being busy, and feel a sense of achievement when dealing with a large workload. Others may experience stress and anxiety under the same conditions, and thrive with a lack of pressure and available time. The critical point here is that individual's response to MWL for a given task is just that: individual, rather than simply assume that high MWL is bad for everybody, there is potential, assuming MWL can reasonably be measured in real time, to dynamically tailor tasks to an individual's best case. If not, then at least it currently asserted in

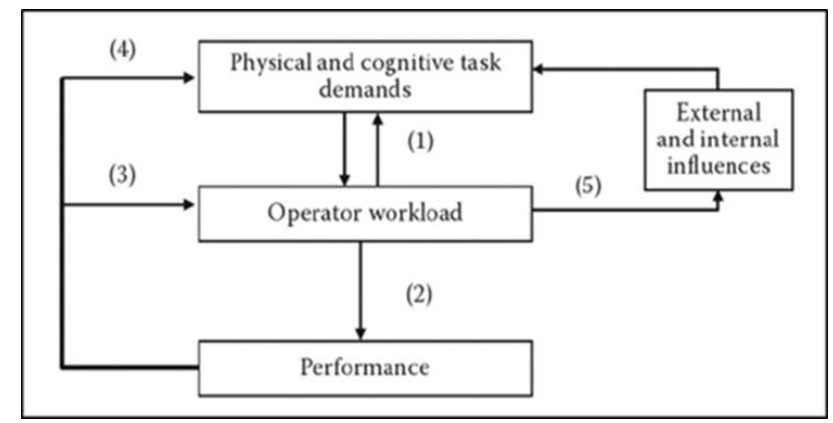

Figure 1: A Framework for MWL definition and evaluation (Wickens, 2008) literature that at least the task context needs to be considered before determining what level of mental workload is 'desirable' [48].

\section{A Mental Workload Measurement Framework}

In order to gain better understanding of the measurement process of MWL, the following MWL framework by [52] might be useful. It comprises three main parts: 1) the physical and cognitive task demands, 2) the operator's workload and 3) the performance (See Figure 1). The external and internal influences on workload and the relationships between the three components are important for MWL definition and measurement.

Physical and cognitive task demands are a reflection of a task's features that are imposed on a person. It is necessary to determine the work demands that individuals might face in order to measure Mental Workload. In addition, because the demand could be perceived differently by different individuals, it is essential to measure both the imposed demands and perceived demand.

Operator workload can be defined as "equivalent to measures of operator strain or effort" [40]. Several MWL measurements focus only on measuring the operator experience during and after the task using different subjective questionnaires, however, it has used the measures of effort from behaviour indices and the impact of effort physiology.

Performance points to measurements that are usually described in terms of speed and errors rates. Nevertheless, measuring performance can be difficult as task complexity increases, as can analysing the relationship between the MWL components (demand, workload and performance) - e.g. "contrary to what is expected, as task demands increase there is not necessarily an increase in operator workload, or decrease in task performance" [40]

\subsection{Stress}

According to [28], they indicated that the meaning of the term "stress" has no precise consensus. It has been defined the "Stress" as the lack of balancing between environmental demands and individual capabilities [30]. It has been suggested that the stress is the interaction between internal or external inverse effects that could challenge or threaten the maintenance of a human's dynamic balance [12]. In other words, stress could indicate to the coping with the external or internal adverse conditions (stressors), which is the behavioural, psychological and physiological responses [12]. It has been indicated that the techniques for inducing the stress response by the body is a status to be unpredictable, and/ or explained as being unprecedented, and/or the person does not the control over this particular situation [33].

Some research refers to "Stress" as a person's experience of pressures and individual demands, whereas the terminology "Strain" indicates to the to the reaction to such stress. However, some researchers have defined the term of stress as non-equivalent degree between demands made upon a person and his/her ability of coping with those certain demands. Also, The European 
Commission Directorate-General for Employment and Social Affairs (2003) described it as a "state characterized by high levels of arousal and distress and often feelings of not coping". According to [30], people will react with stress responses if there is a situation of constant attentional demand. From this point of view, it has been revealed that the psychological stress is the consequence of individuals' appraisals of their environment as exacting or exceeding their resources and/or hazard their safety [30]. The estimates of demanding or threatening surrounding environments are multifaceted, reflecting emotional (affect), motivational (conation), and cognitive processes. However, across nearly all of these dimensions, the notion of 'too much workload' recurs.

The Transactional Model of Stress and Coping (TTSC) [30] , has influenced psychological stress and coping research cross several fields. This theory can be defined as 'a framework which emphasizes appraisal to evaluate harm, threat and challenges, which results in the process of coping with stressful events' [30] . Furthermore, it has been argued that the experienced level of stress, such as emotions, feelings and behaviors because of external stressors, relies upon evaluations of the circumstance, which includes a judgment about whether external demands or internal ones have overtaken the available resources and the ability to cope when demands that surpass resources[30]. In addition, it has been suggested that one definition of stress is that 'the demands of an event exceed personal resources and is motivated by the emotional response to harm and threat' and, in short, is 'the relationship between the person and the environment' [30]. There are two main functions of the appraisal: 1) for modifying the relationship of person-environment and 2) for having an impact on levels of emotional distress. There is a comparison between the cognitive and behavioral efforts required to administer the internal or external environment when there is discord in the individual's ability and resources to deal with psychological stress.

Stress is often perceived in a negative concept while it is actually a very useful and highly adaptive response. Our body understands the importance of stress and how it can cause some of the potential damage, such as insomnia, weakened immune systems, increased blood pressure [6] . In addition, it has been suggested that Psychological stress influences numerous psychological and physical processes in healthy individuals and those with psychiatric disorders, and it is thought to influence mood, memory, and decision-making. Interestingly, such stress can be captured, in a way, by using NASA-TLX (for Mental Workload), which has Frustration as a subscale.

Researchers have applied different subjective and objective measurements to detect and measure psychological stress. Various physiological measurements have been approved in the laboratory experiments as effective measures of stress, such as HR. Interestingly, there are some earlier studies which revealed that induction in Mental Stress can be measured by Skin Temperature [27] , Electrodermal Activity (EDA) [7], HR [7] [26] and Electroencephalography (EEG) with fNIRS [2]. Moreover, there are different research that have investigated stress in real-world experiment by applying various subjective measures, such as SSSQ [22] and Dundee Stress State Questionnaire (DSSQ) [37]

\section{Stress and MWL}

In the Transactional Theory of Stress introduced down [13], the Cognitive appraisal of stress has two different processes: 1) primary appraisal which includes the location of a event, such as stressful, and 2) a secondary appraisal appears after an evaluation of the event, such as threat or challenge. According to the theory of transactions, stress can be occurred in situation of particular transaction is evaluated by individuals relating to their well-being. In other words, the people will face stress when there is perceived mismatch between task's or event's demands and individual's resources to cope with it.

There is similarity between the Transactional Theory of Stress by [14] and the Framework for MWL by [51]. In MWL framework, the operator workload is influenced by the way that task is perceived by the operator, while there is association between demand and performance. It has been pointed out that the outcome demand created by a combination of physical and cognitive task demands and external and internal influences [42]. The workload is affected by intrinsic factors, such as the attitude towards a task and operator skills [42]. These factors could affect the operator decision towards performing a task.

It can be challenging to detect the differences in the way an individual is working to maintain a good level of performance during complex or real-world performance measurements [38]. The results from performance could affect task demands, high demand on the operator could increase the errors that may occur which will lead to increase in the task demands. In the Transactional model, the cognitive appraisal that caused the stress response is influenced by perceived demand and perceived capability. Therefore, if an individual has high capacity to respond to an increased demand, he/she will be less likely to experience a stress response and indeed any stress response could be both

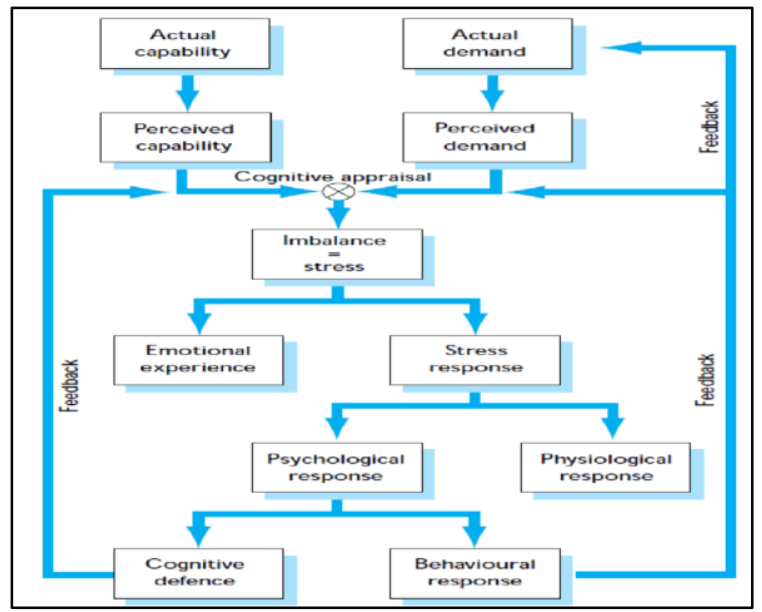

Figure 2: The Transactional Model of Stress (Cox, 1978), updated by (Owusu \& Tawiah, 2014) 
psychological and physiological. When we consider these models together then, it appears to be the case that stress and MWL are strongly interconnected.

\subsection{Measuring MWL and Stress}

There are various subjective methods that are commonly used to assess the impact of psychological stress and mental workload on performance, such as the NASA Task Load Index (NASA TLX) [21] and the Short Stress State Questionnaire (SSSQ) [22]. It has been indicated that such stressors could increase the mental (cognitive) workload significantly [20]. Firstly, a number of studies that highly connected the aroused stress with reducing the capabilities of decision-making and lower performance [23] which could have a negative effect on person's ability. It has been argued that task performance could be reduced by stress which influences individual's concentration and time [39].

In addition, a great number of studies of the psychophysiological measurements have been conducted to conclude the level of workload and stress from measurements of the Autonomic Nervous System (ANS) activity. For example, it has been revealed that the levels of task difficulty during a simulated flight scenario affected both the heart rate (HR) and blood pressure [50]. Moreover, several researchers found that the MWL which assessed by the NASA TLX was significantly related to the HR [53].

Various studies have attempted to measure the stress and MWL, which can be divided into three categories: (1) Self-reporting and subjective ratings using standard questionnaires such as the NASATLX [21] and the SSSQ [22]) ; (2) Behavioral measures, such as primary and secondary-task performance; and (3) physiological measurements, such as including Heart Rate, Skin Temperature to monitor task demand (MW) [8] [20] [50] as well as stress level [9] [14], pupillometry, electromyography, galvanic skin response, and brain activity [51] [53].

Several experiments have been examined the stress and MWL. For example, it has been found that there are significant correlation stress and mental workload (positive correlation) which means when the subjects have higher mental workload and more negative emotion, they tend to experience more stress [55]. In addition, it has been found that stress is a human state which can be caused by a number of reasons, including high mental workload, emotions, or environmental influences [19]. The stress is identified based on the combinations of the recognized positive or negative emotion and the levels of mental workload. Similarly, it has been revealed that there is tight link between mental workload and psychological stress during flight task, where there found a significant positive correlation between HR and mental workload/psychological stress [11].

\section{Conclusion}

To conclude, various research has indicated that MWL and stress are interconnected, and their measures are influenced by each other
[56] [46]. This paper discusses the similar theories of MWL and Stress and outline how these two are connected.

Although a large number of experiments have showed that MIST was worked efficiently and effectively in terms of inducing stress level, our studies revealed that MIST increased experienced mental workload as well as stress. Indeed, its mechanism relies heavily on increasing time pressure in task demand, and this thus manipulating what one would expect to increase mental workload. Our recent results showed that performance will be decreased as the cognitive demands increased which in turn imposed high demands on the operator (The Framework for MWL [51]). Similar to performance, the lack of copying with the perceived demand and perceived capability will impact the cognitive appraisal, which in turn will induce the stress response, that could be psychological and/or physiological [13] . Therefore, we conclude that stress and MWL are highly connected and the participants' experiences of stress should be considered when evaluating MWL. For DigiStress 2019, we recommend that we consider in detail what cognitive factors are changing when we try to 'measure stress', and consider how we model how those different factors affect each other.

An open question, for example, is how Stress and anxiety are similarly connected. Some initial research revealed that here is a relationship between stress and anxiety [34], despite having different definitions and experiences. Stress is associated with frustration and nervousness whereas anxiety is related to fear, worry and unease. Similar to Stress, there are several studies focused on understanding how long-term anxiety could affect mental health and well-being [8][20]. In addition, there are several self-reporting that applied to measure long-term anxiety, such as the Depression Anxiety Stress Scales (DASS) by [29]. However, in our research, it has been concentrated on the anticipation imminent forms of anxiety that focuses on the instantaneous anxiety about imminent performance, such as the Sport Anxiety Scale Questionnaire 2 (SAS-2) (introduced by [45]). To conclude, stress and anxiety have tight similarity and it has been suggested that anxiety is one element of stress experience [15]; yet, we could not find an anxiety theory about that related to our research.

\subsection{Future Work}

Our ongoing and future work is focusing on evaluating different approaches to manipulating experiences of stress, which do not rely on manipulating the task demand, but instead manipulate another facet in the cognitive appraisal. Such a facet might be social performance anxiety from speaking in public [3] [17] (in regard to what others think, rather than in the task being more time pressured), or indeed in anticipation. There are various ways to induce stress, such as time pressure, negative feedbacks, public speech, difficult arithmetical tasks, social comparison, the Stroop Colour Word Test [16], the Sing a Song Stress Test [7] and the Trier Social Stress Test [28]. For our next study, it is necessarily to test these methods to be able to manipulate stress and MWL separately. 
An alternative approach we are considering is to capture participant's experiences of MWL and Stress using a questionnaire, to categorise their response to difficult tasks - whether they found it enjoyable or stressful (similar to [11]). By applying this technique, participants can be divided into different groups by comparing their data based on their experiences, rather than trying to manipulate stress in a within-participants design. Our initial questionnaire to categorize participants will be based upon a simple 5-point scale, unless a more appropriate validated scale is found. This method would be beneficial to compare between groups and help us to understand more about stress and MWL, however comparisons between different people's physiological responses is often difficult, and so reasonable comparison would be hard to establish.

\section{REFERENCES}

[1] Afergan, D., Peck, E. M., Solovey, E. T., Jenkins, A., Hincks, S. W., Brown, Eli T. R. C. \& Jacob, R. JK. (2014).” Dynamic difficulty using brain metrics of workload". In Proc. SIGCHI. ACM, 3797-380

[2] Al-shargie, F., Tang, T. \& Kiguchi, M. (2017). "Assessment of mental stress effects on prefrontal cortical activities using canonical correlation analysis: an fNIRS-EEG study “. Biomedical Optics Express 2583.

[3] Anke, W. B., Marcia, J.W. K., Anne, C. M. \& Michiel, P. W.(2009). "The relation between public speaking anxiety and social anxiety: A review". Journal of Anxiety Disorders. Volume 23, Issue 3, Pages 305-313

[4] Bach, D.R., et al. (2009). "Altered lateralisation of emotional prosody processing in schizophrenia. Schizophrenia Research". 110: 180-187.

[5] Batmaz, I \& Ozturk M. (2008)"Using Pupil Diameter Changes for Measuring Mental Workload under Mental Processing". Journal of Applied Sciences. Volume 8 (1): 68-76.

[6] Baum, A. and Polsusnzy, D (1999.)" Health psychology: mapping biobehavioral contributions to health and illness", Annual Review of Psychology, vol. 50, pp. 137-163.

[7] Brouwer, A. \& Hogervorst, M. A. (2014). "A new paradigm to induce mental stress: the Sing-a-Song Stress Test (SSST)". Front. Neurosci., 29 July 2014 | https://doi.org/10.3389/fnins.2014.00224

[8] Brown, T.A., Brown, L.A., Campbell, C.L., Lehman, J.R., Grisham, R.B. \& Mancill, (2001). "Current and lifetime comorbidity of the DSM-IV anxiety and mood disorders in a large clinical sample". Journal of Abnormal Psychologypp. 585-599

[9] Bonner, M. A. \& Wilson, G. F. (2001). "Heart rate measures of flight test and evaluation,” Int. J. Aviat. Psychol., vol. 12, no. 1, pp. 63-77.

[10] Boucsein, W. (1992). "Electrodermal Activity". New York, NY: Plenum

[11] Calvin, O. K.L. \& Vincent, D. G. (2007) "Development of a facial skin temperature-based methodology for non-intrusive mental workload measurement". Occupational Ergonomics, vol. 7, no. 2, pp. 83-94.

[12] Causse, M., Dehais, F., Faaland, P.\& Cauchard, F.(2012). "An analysis of mental workload and psychological stress in pilots during actual flight using heart rate and subjective measurements". The 5th International Conference on Research in Air Transportation (ICRAT)

[13] Chrousos, P. G. (2009). "Stress and disorders of the stress system". Nature Reviews Endocrinology volume 5, pages 374-381

[14] Cox, T. (1978). Stress. London: MacMillan Press.

[15] Dobkin PL. \& Pihl, RO. (1992) "Measurement of psychological and heart rate reactivity to stress in the real world." Psychother Psychosom.58(3-4):208-14.

[16] Donald, W. P. \& Marian, J. (2017). "Hormones, Brain and Behavior". Third Edition. Elsevier Inc. All rights reserved.

[17] Douchamps, J. (1988). "A brief, versatile, computerized, stress-inducing task derived from the Stroop color word test." https://www.ncbi.nlm.nih.gov/pubmed/3226226

[18] Gerlinde, C. H., Winnie, E., Talia, Z. \& Richard, G. H. (2003). "Behavioral assessment of public-speaking anxiety using a modified version of the Socia Performance Rating Scale". Behaviour Research and Therapy. Volume 41, Issue 11, Pages 1373-1380

[19] Gjoreski, M., Luštrek, M., G ams, M., \& Gjoreski, H. (2017). "Monitoring stress with a wrist device using context". J. Biomed. Inform. 73: 159-170.

[20] Gunnar, M. \& Fisher, P. (2006).'The Early Experience, Stress, and Prevention Network. Bringing basic research on early experience and stress neurobiology to bear on preventative interventions for neglected and maltreated children". Development and Psychopathology. 18:651-677.

[21] Hagmüller, M., Rank, E. \& Kubin, G. (2006)." Evaluation of the Human Voice for Indications of Workload-induced Stress in the Aviation Environment". EEC NOTE.
[22] Hankins, T. C., \& Wilson, G. F. (1998). "A comparison of heart rate, eye activity, EEG and subjective measures of pilot mental workload during flight". Aviation, Space, and Environmental Medicine, 69(4), 360-367

[23] Hart, S. G., and Staveland, L. E. (1988).” Development of NASA-TLX (task load index): Results of empirical and theoretical research". Human mental workload.

[24] Helton, W.S., and Näswall, K., (2014) "Short Stress State Questionnaire Factor Structure and State Change Assessment" European Journal of Psychological Assessment.

[25] Helmreich, R., Chidster, T., Foushee, H., Gregorich, S. \& Wilhelm, J. (1990) "How effective is cockpit resource management training? Issues in evaluating the impact of programs to enhance crew coordination," Flight Safety Dig., vol. 9, no. 5, pp. 1-17.

[26] Hindra, K., Alexandr, V. M.\& Mykola, P.(2013). "Stress detection from speech and Galvanic Skin Response signals". Proceedings of the 26th IEEE International Symposium on Computer-Based Medical Systems

[27] Hirshfield, L. M., Solovey, E. T., Girouard, A., Kebinger, J., Jacob, R. J., Sassaroli, A., and Fantini, S. (2009) "Brain measurement for usability testing and adaptive interfaces: an example of uncovering syntactic workload with functional near infrared spectroscopy". In Proc. CHI, ACM, 2185-2194

[28] Jex, S.M., Beehr, T.A., \& Roberts, C.K. (1992). The meaning of occupational stress items to survey respondents. Journal of Applied Psychology, 77, 623-628.

[28] Julian F. Thayer, Fredrik Åhs, Mats Fredrikson, John J. Sollers III, Tor D. Wager (2012) "A meta-analysis of heart rate variability and neuroimaging studies: Implications for heart rate variability as a marker of stress and health “" Neuroscience and Biobehavioural Reviews. Volume 36, Issue 2, Pages 747-756.

[29] Katherine A. Herborn, James L. Graves, Paul Jerem, Neil P. Evans, Rued Nager,1 Dominic J. McCafferty,1 and Dorothy E.F. McKeegan1(2015) "Skin temperature reveals the intensity of acute stress" Physiol Behav. 152(PtA): 225230

[30] Kirschbaum, C., Pirke, K.-M. \& Hellhammer, D.H. (1993)." The 'Trier Socia Stress Test' - A Tool for Investigating Psychobiological Stress Responses in Laboratory Setting". Neuropsychobiology ;28:76-81.

[29] Lovibond, P.F., and Lovibond, S.H. (1995). "The structure of negative emotional states: Comparison of the Depression Anxiety Stress Scales (DASS) with the Beck Depression and Anxiety Inventories". Behaviour Research and Therapy. 33, 3: 335-343

[30] Lazarus, R.S., \& Folkman, S. (1984). "Stress, appraisal and coping". New York: Springer.

[31] Leanne, M. \& Robert, J. (2009).” Using Brain Measurement to Evaluate Reality Based Interactions". Challenges in the Evaluation of Usability and User Experience in Reality Based Interaction 5, 19-20.

[32] Lukanov, K. E., Maior, H. A., \& Wilson, M. L. 2016. Using fNIRS in usability testing: understanding the effect of web form layout on mental workload. In: Proc. CHI'16. 4011-4016.

[33] Lupien, S. J., McEwen, B. S., Gunnar, M. R., \& Heim, C. (2009). "Effects of stress throughout the lifespan on the brain, behaviour and cognition". Nat Rev Neurosci, 10(6).

[34] Maes, M., Song, C., Lin, A., De Jongh, R., Van Gastel, A., Kenis, G., Bosmans, E., De Meester, I., Benoy, I., Neels, H., Demedts, P., Janca, A., charpé, S. \& Smith, RS. 1998. The Effects of Psychological Stress on Humans: Increased Production of Pro-Inflammatory Cytokines and Th1-Like Response In StressInduced Anxiety. Cytokine. 10, 4: 313-318

[35] Maior, H., Pike, M., Sharples, S., \& Wilson, M. L. 2015. Examining the Reliability of Using fNIRS in Realistic HCI Settings for Spatial and Verbal Tasks. In Proc. CHI'15. 3039-3042.

[36] Maior, H. A., Wilson, M. L., and Sharples, S., 2018. Workload Alerts-Using Physiological Measures of Mental Workload to Provide Feedback During Tasks. ACM Trans.

[37] Matthews, G., Szalma, J., Panganiban, A., Neubauer, C., and Warm, J. S., (2013). "Profiling task stress with the dundee stress state questionnaire". Psychology of stress: New research. 49-90.

[38] Megaw, T. \& Sharples, S. (2015). "The definition and measurement of mental workload. Evaluation of human work, Eds. Esmond N". Corlett, and John R. Wilson (525-551

[39] Muse, L.A., Harris, S.G. \& Field, H.S. (2003). "Has the inverted-U theory of stress and job performance had a fair test?". Human Performance. 16, 349-364.

[40] Nomikos, M. S., Opton, E., Jr., \& Averill, J. R. (1968). Surprise versus suspense in the production of stress reaction. Journal of Personality and Social Psychology, 8(2, Pt.1), 204-208.

[41] Owusu, G. A. \& Tawiah, M. A. (2014). "Stress Management among Senior Staff Female Administrators in the University of Cape Coast". International Journal of Academic Research in Progressive Education and Development. Vol. 3, No. 4. ISSN: 2226-6348

[42] Peck, E. M., Yuksel, B. F., Ottley, A., Jacob, R. J., and Chang, R. (2013) “Using fNIRS Brain Sensing to Evaluate Information Visualization Interfaces". In CHI, $\mathrm{ACM}$ 
[43] Pickup, L., Wilson, J.R., Norris, B.J., Mitchell, L., Morrisroe, G., 2005. The integrated workload scale (IWS): a new self-report tool to assess railway signaller workload. Appl. Ergon. 36 (6), 681e693.

[44] Ravenswaaij et al., C. M. A. van. (1993).“Heart rate variability,” Ann. Int. Med., vol. 118, no. 6, pp. 436-447, 1993.

[45] Ronald, E., Smith, F. L., Smoll, S. P., Cumming, \& Joel, R. G. (2006)" Measurement of Multidimensional Sport Performance Anxiety in Children and Adults: The Sport Anxiety Scale-2". Journal of Sport \& Exercise Psychology. 28, 479-501

[46] Sanders, A. F. (1983). "Towards a model for stress and human performance". Acta Psychologica, 53, 61-97.

[47] Selye, H. 1936. A syndrome produced by diverse nocuous agents. Nature. 138 (1), 32

[48] Sharples, S. (2019). "Workload II: A Future Paradigm for Analysis and Measurement: Volume VII: Ergonomics in Design, Design for All, Activity Theories for Work Analysis and Design, Affective Design". Proceedings of the 20th Congress of the International Ergonomics Association (IEA 2018), pp.489498

[49] Smith, R.E., Smoll, F.L., \& Schutz, R.W. (1990). "Measurement and correlates of sport-specific cognitive and somatic trait anxiety: The Sport Anxiety Scale". Anxiety Research, 2, 263-280

[50] Veltman, J. A., \& Gaillard, A. W. (1996). "Physiological indices of workload in a simulated flight task". Biological Psychology, 42(3), 323-342.

[51] Wickens, C. D. (2008)." Multiple resources and mental workload". The Journal of the Human Factors and Ergonomics Society 50, 3 (2008), 449-455

[52] Wilson, J. R \& Sharples, S. (2015). "Evaluation of human work". CRC Press. 521-522 pages.

[53] Wilson, G. (2002). "An analysis of mental workload in pilots during flight using multiple psychophysiological measures". The International Journal of Aviation Psychology, 12(1), 3-18.

[54] Xie, B., and Salvendy, G. (2000). Review and reappraisal of modelling and predicting mental workload in single- and multi-task environments. Work Stress 14, 74-99. doi: 10.1080/026783700417249

[55] Xiyuan H., Yisi L., Olga S., Yun R. E. T., Lipo W., Wolfgang M. W. (2015)" EEG Based Stress Monitoring”. IEEE International Conference on Systems.

[56] Yerkes, R. M. \& Dodson, J. D. (1908)." The Relation Of Strength Of Stimulus To Rapidity Of Habit-Formation". Journal of Comparative Neurology and Psychology, 18, 459-482

[57] Yuksel, B. F., Oleson, K. B., Harrison, L., Peck, E. M., Afergan, D., Chang, R., \& Jacob, R. J., (2016). "Learn piano with BACh: An adaptive learning interface that adjusts task difficulty based on brain state”. In Proc. CHI'16. 5372-5384. 